\title{
Importance of Hydration and Surface Structure for Friction of Acrylamide Hydrogels
}

\author{
Rok Simič ${ }^{1}$ Melis Yetkin ${ }^{1} \cdot$ Kaihuan Zhang ${ }^{1} \cdot$ Nicholas D. Spencer $^{1}$
}

Received: 27 February 2020 / Accepted: 8 April 2020 / Published online: 23 April 2020

(c) The Author(s) 2020

\begin{abstract}
To understand the dissipative mechanisms in soft hydrogel lubrication, polyacrylamide (PAAm) hydrogels with two distinct surface structures were examined under various contact conditions. The characteristic speed-dependent friction of the selfmated, crosslinked hydrogel surfaces could be explained by hydrodynamic shearing of a thin water layer between two rather impermeable bodies. On the other hand, the frictional response of brushy hydrogel surfaces is dependent on the contact conditions and the level of surface hydration. In a migrating contact, brushy hydrogels showed low, speed-independent friction $(\mu \sim 0.01)$ likely due to a thick layer of shearing liquid trapped within the sparse surface network. In stationary contact, however, brushy hydrogel surfaces can partially exude water from the near-surface region over time, as shown by timeresolved Fourier-transform infrared (FTIR) spectroscopy. This is assumed to be reflected in a friction increase over time. Interfacial shearing appears to shorten the characteristic exudation times compared to those observed under static loading. Once fluid has been exuded, brushy surfaces were shown to reach similar friction values as their crosslinked analogs. The results thus indicate that the dominating dissipation mechanism during sliding at low contact pressures is shearing of the interfacial liquid film, rather than poro-elastic dissipation within the bulk. Maintenance of surface hydration is therefore crucial, in order to take advantage of the low friction of such systems.
\end{abstract}

Keywords Hydrogels $\cdot$ Biotribology $\cdot$ Aqueous lubrication $\cdot$ Friction $\cdot$ Hydration $\cdot$ Exudation

\section{Introduction}

Surfaces of biological tissues at sliding interfaces, such as the cornea and eyelid or articular cartilage, are soft, permeable materials covered by brushy, water-rich mucous structures that provide low shear stress on the underlying tissue over a wide range of sliding speeds and contact stresses [1-3]. Synthetic hydrogels, on the other hand, are polymer networks rich in interstitial water and, due to their structure, are promising candidates to mimic the lubrication mechanisms of the above-mentioned tissues. The good lubrication properties of hydrogels are often associated with their high

Electronic supplementary material The online version of this article (https://doi.org/10.1007/s11249-020-01304-x) contains supplementary material, which is available to authorized users.

Nicholas D. Spencer

nspencer@ethz.ch

1 Laboratory for Surface Science and Technology, Department of Materials, ETH Zürich, Zurich, Switzerland water content, high water permeability, low elastic modulus, and their ability to form a water film at the sliding interface [4].

However, these good lubrication characteristics of hydrogels and similar biphasic materials have long intrigued researchers, and several mechanisms have been proposed to explain the origin of the frictional dissipation in such systems. Gong et al. observed that hydrogel friction does not conform to Amonton's law $F=\mu F_{\mathrm{n}}$, where $F$ is the friction force, $F_{\mathrm{n}}$ is the normal load, and $\mu$ the coefficient of friction [5]. Instead, they observed little dependence on the load and rather a significant dependence on the sliding speed, which they attributed to the very strong hydration ability of the gel, which makes it possible to sustain hydrodynamic lubrication, even at low sliding velocity and relatively high contact pressure. For gels sliding over a solid substrate, Gong proposed the adsorption-repulsion model, which depends on the interaction between the gel and the substrate $[6,7]$. As an attractive interaction, the elastic stretching and detaching of the adsorbed polymer chains during relative motion were proposed to account for the frictional force. For the repulsive 
(or nonadhesive) interaction, however, the viscous flow of the interfacial solvent was suggested to dictate the frictional force. Starting with Newton's law for viscous flow and taking into account de Gennes' scaling concepts from polymer physics [8], Gong proposed the following shear-stress proportionality for the case of the contact pressures $P$ that are lower than the osmotic pressure $\Pi$ and the elastic modulus $E$ of the gel $(P<\Pi \approx E)$ :

$\frac{F}{A}=\tau \propto \frac{\eta \nu P}{c^{3 / 2}}$

where $A$ is the contact area, $\tau$ is the shear stress, $\eta$ is solvent viscosity, $v$ is the relative sliding speed, $P$ is the contact pressure due to the normal load, and $c$ is the polymer concentration [6]. Interestingly, the given expression shows a linear dependence of friction force on load, and also predicts a decrease of the frictional force with an increase of the polymer concentration, presumably due to the stronger interfacial repulsion and hence a thicker interfacial solvent layer, which is contrary to recent observations made by the group of Sawyer [4, 9]. Although the equation also proposes linear proportionality to the sliding speed $(F \propto v)$, their experimental results show power-law scaling with exponents between 0.21 and 0.67 , which they reason could be due to an overestimation of the hydrodynamic friction. They state that the considered no-slip boundary might actually be located within the material due to solvent permeability and that the flow within the thin ( few nm thick) shearing layer might be non-Newtonian [6]. However, no hydrodynamically induced film thickness increase was considered that might potentially lower the power exponent below unity.

Using the Debye-Brinkman equation, Gong and coworkers took into account the flow within the gel, proposing the following expression for the shear stress $\tau$ between two similar hydrogel surfaces [10]:

$\tau \propto \frac{\eta v}{2 l+2 \sqrt{K}}$

where $2 l$ is the gel surface separation and $K$ is the permeability of the hydrogel. They assumed that shear flow can penetrate the gel up to a depth of $\sqrt{K}$, which should be on the order of the mesh size $\xi$. The hydrodynamic thickness $h=2 l+2 \sqrt{K}$ in the case of self-mated, nonadhesive gels depends on the repulsion between the sliding surfaces, and hence, on the amount of charge at the gel surface [10]. According to these authors, however, the proposed equation underestimates the coefficient of friction by an order in magnitude compared to experimentally obtained coefficients of friction, which could be due to the existence of "bound water" in the proximity of the polymer moieties [10]. Applying the above equation to the experimentally measured friction of self-mated polysaccharide gels returned hydrodynamic thicknesses of 30-300 nm for charged gels [11]. However, the thickness of 5-20 nm obtained for neutral gels seemed too low for effective hydrodynamic lubrication to take place. Therefore, using the bulk viscosity value of water apparently underestimates the friction, and considering an effective viscosity of water was suggested [11]. Despite that, the theoretical prediction qualitatively demonstrates the hydrodynamic nature of the friction between gel surfaces.

Although the initial theoretical predictions suggested linear proportionality between the friction force $F$ and sliding speed $v$, Kagata et al. later showed that friction force between two repulsive hydrogel surfaces follows a power law, $F \propto v^{\mathrm{n}}$ [12]. The exponent $n$ was almost zero at low contact pressures between self-mated hydrogels and was increasing with increasing contact pressure until it saturated at about 0.55 . They could not explain the observed behavior, but non-Newtonian behavior of the interfacial water and the viscoelastic nature of the gel were proposed as possible contributing factors [12]. It was also observed that friction does not disappear at very low sliding speeds, as suggested by the above expression, even for a repulsive contact of selfmated charged hydrogels. Instead, the friction leveled off at low sliding speeds and was almost independent of speed. Moreover, Kagata et al. reported a significant static friction upon initiation of sliding, indicating a non-hydrodynamic dissipation mechanism at very low sliding speeds [13].

A similar transition from a low, speed-independent friction at low sliding speeds to a speed-dependent friction that follows a power law with an exponent of 0.5 was also observed by the group of Sawyer $[9,14]$. They termed the speed-independent regime as thermal-fluctuation lubrication. According to the authors, the scaling of friction within the speed-dependent regime $\left(F \propto v^{0.5}\right)$ could be predicted by a hydrodynamic lubrication theory, but the predicted friction coefficient would be over an order of magnitude lower than the measured values. Predictions assuming viscoelastic dissipation yielded frictional forces that were also several orders of magnitude below those measured. The exact mechanism seemed to be unclear, but the authors proposed that at high sliding speeds, the polymer relaxation time $t_{\mathrm{r}}=\xi^{3} \eta / k_{\mathrm{b}} T$ becomes longer than the competing timescale acting against the polymer relaxation $\left(t_{\mathrm{r}}>t=v / \xi\right)$, and thereby coined the term polymer-relaxation lubrication for this regime.

Contrary to the hydrodynamic theory, Delavoipière et al. proposed that most of the frictional-energy dissipation comes from the poro-elastic flow near the sliding contact, while they assumed no significant contribution from the interfacial hydrodynamic dissipation [15, 16]. However, their experiments were performed on thin $(\sim 15 \mu \mathrm{m})$ hydrogel layers confined between a glass sphere and a flat glass substrate, resulting in contact pressures above $1 \mathrm{MPa}$, which presumably exceed the osmotic pressure and hence caused 
significant poro-elastic fluid migration. Therefore, for nonadhesive, self-mated hydrogels sliding at contact pressures below the value of their osmotic pressure ( elastic modulus), it seems that the frictional dissipation has an interfacial origin rather than resulting from visco- or poro-elastic dissipation.

We have recently confirmed the observation of Gong, that the nature of hydrogel friction depends on the hydrogel surface structure, which is strongly dependent on the way in which the gel is molded $[17,18]$. Crosslinked hydrogel surfaces showed a speed-dependent friction following a power law with an exponent of about 0.5 , whereas a similar hydrogel with a sparser, brushier surface showed lower and almost speed-independent friction over the same range of sliding speeds [18]. We have proposed a frictional mechanism based on the hydrodynamic shearing of interfacial water. Crosslinked hydrogel surfaces seemed to behave as if they were almost impermeable, where the friction was dictated by the hydrodynamic shearing of a thin, nanoscopic water layer. If the thickness of this thin layer increases proportionally to the square-root of the sliding speed, due to the elasto-hydrodynamically formed wedge in the contact, the friction force should scale as $F \propto v / h=v / v^{0.5}=v^{0.5}$ [19]. Experimentally observed film thicknesses for a sphere-onflat geometry were found to scale as $h=v^{0.57}$ [19]. For the case of an isoviscous-elastic contact, Hamrock and Dowson proposed scaling of $h=v^{0.65}$ [20]. Assuming the thickness scaling from both these cases would result in a friction force that scales as $F \propto v^{0.35-0.43}$, which would underestimate the scaling observed in our previous work. However, the discrepancy could arise from the geometrical differences between a ball-on-flat and a flat-on-flat contact. Nevertheless, it was observed that the presence of a convergent zone ahead of the contact area can substantially enhance hydrodynamic pressurization and contact rehydration, which, in turn, reduces friction [21].

For the brushy, hydrogel surfaces, however, the water shearing within the sparse, brushy surface structure seems to create a thicker hydrodynamic film, which results in a significant drop in friction compared to the crosslinked surfaces [18]. Moreover, the friction between the brushy hydrogel surfaces shows only a weak speed dependency, presumably due to an interplay between water exudation under load and hydrodynamically induced layer thickening.

In order to clarify the dissipation mechanisms behind friction and elucidate the behavior of hydrated surface layers, we have synthesized hydrogels with crosslinked and brushy surfaces by molding them against different molding materials. We have characterized their surfaces by means of colloidal-probe nanoindentation in liquid. The hydrogels were then tested in various contact geometries, under unidirectional and reciprocating sliding, as well as in self-mated or mixed contact pairs. We have compared the results of the time-resolved ATR-FTIR experiments with the observed time evolution in the coefficient of friction, in order to confirm the hydrodynamic origin of friction and the importance of the surface structure and sufficient surface layer hydration for maintaining a thick shearing fluid film and low coefficient of friction.

\section{Materials and Methods}

\subsection{Materials}

Acrylamide (AAm, Sigma-Aldrich, St. Louis MO, USA, $>99 \%$ ) and $N, N^{\prime}$-methylenebisacrylamide (bisAAm, Sigma-Aldrich, $>99.5 \%$ ) were used as monomer and crosslinker, respectively. Milli-Q ultrapure water was used to dissolve $10 \mathrm{wt} \%$ of the monomer, $0.4 \mathrm{wt} \%$ of the crosslinker, and $0.01 \mathrm{wt} \%$ of lithium phenyl-2,4,6-trimethylbenzoylphosphinate (LAP), the synthesis of which is described in our previous work [22]. The solution was carefully poured into piranha-cleaned glass petri dishes or into sterilized polystyrene (PS) petri dishes to a height of approximately 3-4 $\mathrm{mm}$. The two different molding materials were utilized in order to produce hydrogels with two distinct surfaces, being either dense and crosslinked in the case of the glass mold, or sparse and brushy in the case of the PS mold [18, 23, 24]. The molds, filled with the solution, were polymerized for 20 min under UV light with an intensity of about $1.4 \mathrm{~mW} / \mathrm{cm}^{2}$ at a wavelength of $365 \mathrm{~nm}$ (Stratalinker UV Crosslinker 2400, Stratagene Corp., La Jolla, CA, USA). After polymerization, the gels were demolded and immersed in a copious amount of Milli-Q ultrapure water for at least $48 \mathrm{~h}$ to remove unreacted species and allow swelling of the gels. For the friction and the spectroscopy experiments, the gel samples of the appropriate dimensions were punched out of bigger slabs of already swollen hydrogels. Such hydrogel samples were then attached to steel pins or PS petri dishes using a thin layer of cyanoacrylate-based superglue (Pattex, Henkel AG \& Co. KGaA, Düsseldorf, Germany). All experiments were performed with the hydrogels fully immersed in ultrapure water.

\subsection{Nanoindentation}

An atomic force microscope (AFM, MFP-3D ${ }^{\mathrm{TM}}$, Asylum Research, Santa Barbara, USA) equipped with a liquid cell was used to perform nanoindentation experiments with samples fully immersed in ultrapure water. The normal spring constant $k$ of the gold-coated tipless cantilever (NSC-36, Mikromash, Estonia) was determined by the Sader method [25]. A silica microsphere (GP0083, Whitehouse Scientific, Waverton, UK) with a radius of $14 \mu \mathrm{m}$ was attached to the end of the tipless cantilever with a 2-component epoxy 
resin adhesive (UHU GmbH, Germany), and the effective spring constant of the probe was calculated as $k^{\prime}=k$ $\left(L / L^{\prime}\right)^{3}=1.28 \mathrm{~N} / \mathrm{m}$, where $L$ and $L^{\prime}$ are the distances from the base of the cantilever to the tip of the cantilever and to the colloid position, respectively [26]. The optical-lever sensitivity $S$, indicating the relation between the change in cantilever deflection $x$ and the photodiode signal $U$, was calibrated by pressing the probe against a silicon wafer in water. The indentation depth was calculated as $d=Z-x=Z-S U$, where $Z$ is the measured z-piezo displacement. The force was calculated as $F=k^{\prime} x$. The root-mean-square value of the noise away from the surface $\sigma$ was $20-30 \mathrm{pN}$, and the contact point was determined as the last data point lying within $2 \sigma$ from the zero-force line on the approach curve. Indentation speed was set to $1 \mu \mathrm{m} / \mathrm{s}$, with only a minor hysteresis between approach and retraction, indicating the insignificance of the visco- or poro-elasticity contributions. All measurements were performed at room temperature of $25{ }^{\circ} \mathrm{C} \pm 1{ }^{\circ} \mathrm{C}$. An acquisition rate of $2000 \mathrm{~Hz}$ was used. Force maps of $3 \times 4$ force curves were obtained over an area of $40 \times 40 \mu \mathrm{m}^{2}$ on three different locations of a sample to check for reproducibility. Results are presented as representative force-indentation curves.

\subsection{Friction}

Friction of hydrogel-hydrogel pairs was measured using several different contact geometries, Fig. 1. To avoid deformation-related visco- and poro-elastic dissipation during sliding, which could have a significant contribution to the lateral force during sliding of a pin over a soft substrate, unidirectional sliding of a ring on a disc was used (Fig. 1a).
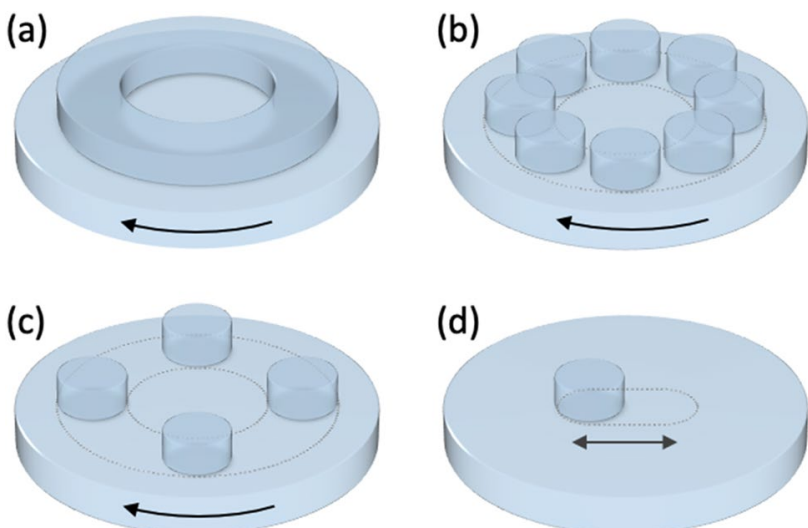

Fig. 1 Schematics of the hydrogel-hydrogel contact configurations used in the friction experiments. Unidirectional sliding of a ring-ondisc, b 8-pins-on-disc, $\mathbf{c}$ 4-pins-on-disc and $\mathbf{d}$ reciprocating sliding of a pin-on-disc was used. The ring had an inner and outer diameter of 24 and $40 \mathrm{~mm}$, respectively. The pins had a diameter of $10 \mathrm{~mm}$. In all cases, the contact pressure was about $6 \mathrm{kPa}$ and the hydrogels were completely immersed in ultrapure water
For this, a ring with the inner and the outer diameter of 24 and $40 \mathrm{~mm}$, respectively, was punched out of a slab of a hydrogel with a thickness of about $4 \mathrm{~mm}$ and glued to the top parallel plate of the rheometer (ARES-G2, TA Instruments, Delaware, USA). A disc with a diameter of $50 \mathrm{~mm}$ was punched out of a similar hydrogel slab and glued to a PS petri dish that was attached to the lower parallel plate of the rheometer and filled with ultrapure water. The normal load was set to $5 \mathrm{~N}$ corresponding to a contact pressure of about $6 \mathrm{kPa}$. To calculate the contact pressure, the contact area was assumed to be equal to the nominal area of the upper specimen, where negligible lateral expansion under load was assumed. Sliding was induced by rotating the lower specimen at angular velocities ranging from 0.005 to $1.0 \mathrm{rad} / \mathrm{s}$. Assuming a constant shear stress over the contact area, the effective radius was calculated to be $16.3 \mathrm{~mm}$. The effective sliding speed was hence in a range from 0.08 to $16.3 \mathrm{~mm} / \mathrm{s}$. The tests were performed from the slowest sliding speed to the fastest and then again gradually back to the slowest, where multiple full rotations were performed at each sliding speed. The effective frictional force was determined from the equilibrated, second half of each test. Coefficients of friction were determined as the ratio of the effective friction force and the normal load. No significant difference was observed between the friction values obtained during the two ramp directions. This procedure was performed three times, each time with another set of hydrogel samples. Friction values of both ramps and all three repetitions were averaged at a given speed and are presented with error bars corresponding to one standard deviation.

To avoid continued compressive and shear stress on the bottom hydrogel surface during the ring-on-disc experiment, discontinuities were introduced by replacing the upper hydrogel ring with either 4 or 8 flat, circular pins, Fig. $1 \mathrm{~b}$ and c. The pins had a diameter of $10 \mathrm{~mm}$ and were positioned with their centers at a radius of $15 \mathrm{~mm}$ from the axis of rotation. The normal load in the case of the 4 and the 8 pins was adjusted to 2 and $4 \mathrm{~N}$, respectively, to match the contact pressure in the ring-on-disc experiment $(6 \mathrm{kPa})$. Using the same angular velocities as in the ring-on-disc case yielded average sliding speeds in the range of $0.08-15 \mathrm{~mm} / \mathrm{s}$. All experiments with the rheometer were performed in a self-mated configuration of the hydrogels (i.e., brushy/ brushy or crosslinked/crosslinked).

Reciprocating sliding experiments were performed using a tribometer (CSM, Needham, MA, USA). Again, a flat, circular hydrogel pin of $10 \mathrm{~mm}$ diameter was pressed against a larger hydrogel disc with a normal load of $0.5 \mathrm{~N}$ to match the contact pressure of $6 \mathrm{kPa}$ from the unidirectional sliding experiments. The stroke length was set to $10 \mathrm{~mm}$ and the sliding speeds in the center of the stroke were varied from 0.1 to $15 \mathrm{~mm} / \mathrm{s}$. The friction loops appeared symmetric with negligible transient effects upon reversal of the sliding 
motion (Fig. S1 in Supporting Information). The friction force was determined from the middle $20 \%$ of the stroke length, where the sliding speed varied by less than $2 \%$. A minimum of 20 cycles were performed for each condition, in order to reach steady state. The last five cycles were considered for determining the characteristic friction for each condition. Characteristic friction values from at least 3 repetitions of each experiment were averaged at a set sliding speed and are presented with error bars that correspond to one standard deviation.

\subsection{Infrared Spectroscopy}

Flat, cylindrical hydrogel pins, $10 \mathrm{~mm}$ in diameter, similar to those used for the friction experiments, were attached to flat-ended, stainless-steel pins and pressed with their crosslinked or brushy surfaces against the diamond crystal of the ATR-FTIR spectrometer (Alpha-P, Bruker, Billerica, MA, USA), Fig. 2. The normal loads applied to the pins were between 0.5 and $2 \mathrm{~N}$, corresponding to a contact pressure of 6- $25 \mathrm{kPa}$ between the hydrogel surface and the ATR crystal. The spectra were recorded from 500 to $4000 \mathrm{~cm}^{-1}$ by averaging 64 scans at a resolution of $4 \mathrm{~cm}^{-1}$ using a DTGS detector. The background was recorded in ultrapure water in

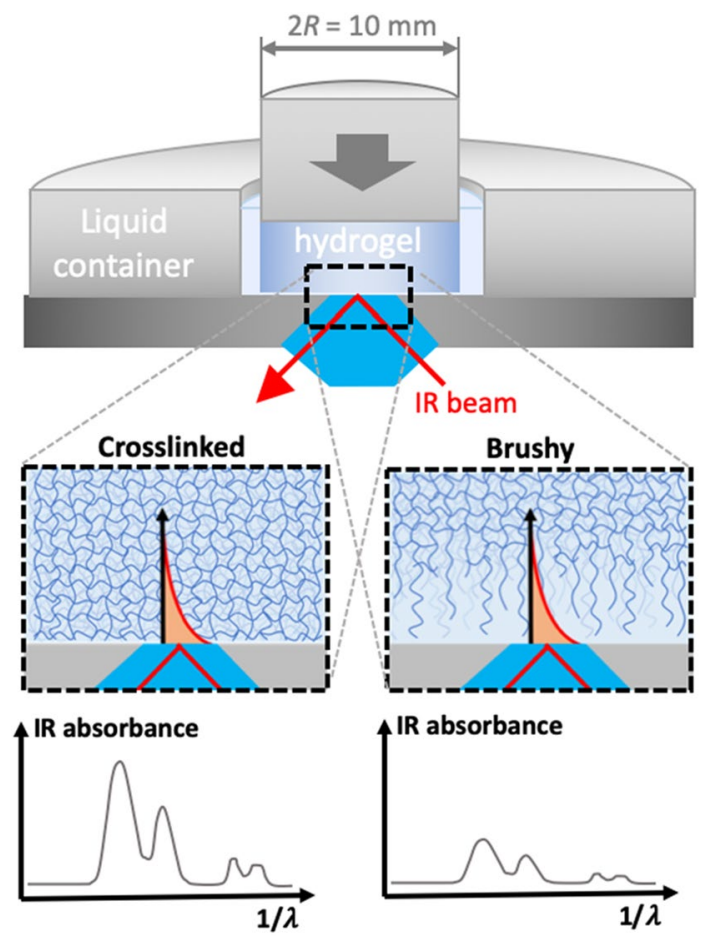

Fig. 2 Schematic cross-section of the ATR-FTIR setup. A flat, circular hydrogel pin was pressed with its crosslinked or brushy surface against a diamond ATR crystal at various loads. The absorbance spectra were recorded as a function of time and the intensity of the characteristic peaks in the IR spectrum that reflect the polymer concentration close to the hydrogel surface were analyzed order to obtain the spectra that correspond to the polymer. As expected, the most pronounced absorption appeared at about $1660 \mathrm{~cm}^{-1}$ and $1610 \mathrm{~cm}^{-1}$, corresponding to the amide I and amide II bands, respectively [27]. The two peaks were fitted using a linear combination of Gaussian and Lorentzian functions. The area under the fit for the amide II band was calculated and followed for both hydrogel gels as a function of time and contact pressure. The intensities for the amide I band only differed in their peak intensities and were qualitatively similar. The analyzed peak intensities correspond to the amount of polymer within the first few microns of a hydrogel surface, with the higher intensity corresponding to the denser polymer network within the near-surface region. The corresponding inverse change in the water $\mathrm{OH}$ peak at $3000-3500 \mathrm{~cm}^{-1}$, however, overlaps with the N-H stretching band of the amide in the same region and was thus not analyzed.

\section{Results and Discussion}

\subsection{Nanoindentation}

Figure 3 shows representative force-indentation curves obtained by nanoindentation of the two hydrogel surfaces. The curves obtained on the surfaces of the glass-molded hydrogels followed a Hertzian contact model, indicating a homogeneous structure with an elastic modulus of $29 \mathrm{kPa} \pm 1 \mathrm{kPa}$. On the other hand, the PS-molded hydrogel showed a much softer initial response, with a progressive increase in the slope of the indentation curve beyond the

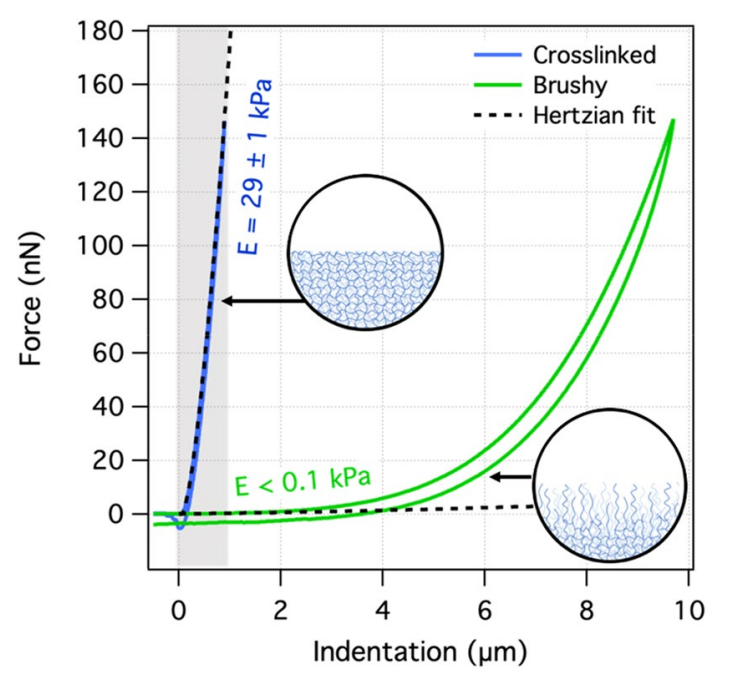

Fig. 3 Force-indentation curves obtained by indentation of the crosslinked and brushy hydrogel surfaces in water. The elastic moduli were determined by fitting a Hertzian contact model to the data within the first $1 \mu \mathrm{m}$ of the indentation depth (shaded gray). Extrapolated Hertzian fits are shown as black dotted lines 
Hertzian regime, as also observed in our recent work [18, 24]. Fitting the Hertzian contact model to the initial $1 \mu \mathrm{m}$ of the indentation depth gives an elastic modulus below $0.1 \mathrm{kPa}$. This indicates a very sparse and presumably brushy surface. However, the exact crosslinker-to-monomer concentration near the surface could not be determined. With increasing indentation, the sparse structure then gradually densifies to a similar density and structure as that observed for the homogenous and crosslinked, glass-molded surface. The evolution of the indentation curves suggests that this soft gradient layer has a thickness on the order of 10-20 $\mu \mathrm{m}$.

\subsection{Friction}

The coefficient of friction as a function of sliding speed for different, self-mated contact configurations during unidirectional sliding is shown in Fig. 4. The coefficient of friction of self-mated, crosslinked hydrogel surfaces in a ring-on-disc configuration increased from about 0.06 at $0.08 \mathrm{~mm} / \mathrm{s}$ to about 0.6 at $16 \mathrm{~mm} / \mathrm{s}$. In contrast to a pin-on-disc configuration, where a hydrogel disc undergoes a migrating deformation, a ring-on-disc configuration eliminates local changes in hydrogel deformation during sliding and should therefore eliminate any poro-elastic contribution to the dissipation. Thus, the observed friction should originate primarily from interfacial shearing. Assuming that the hydrogel surface resembles the surface of the mold, the surface roughness values $R_{\mathrm{a}}$ for the glass-molded hydrogel would be about $1 \mathrm{~nm}$ [18]. Considering the values of coefficient of friction and a bulk-water dynamic viscosity of $1 \mathrm{mPas}$, according to Newton's law of viscous flow, this would result in a hydrodynamic thickness on the order of $1 \mathrm{~nm}$ over the entire range of

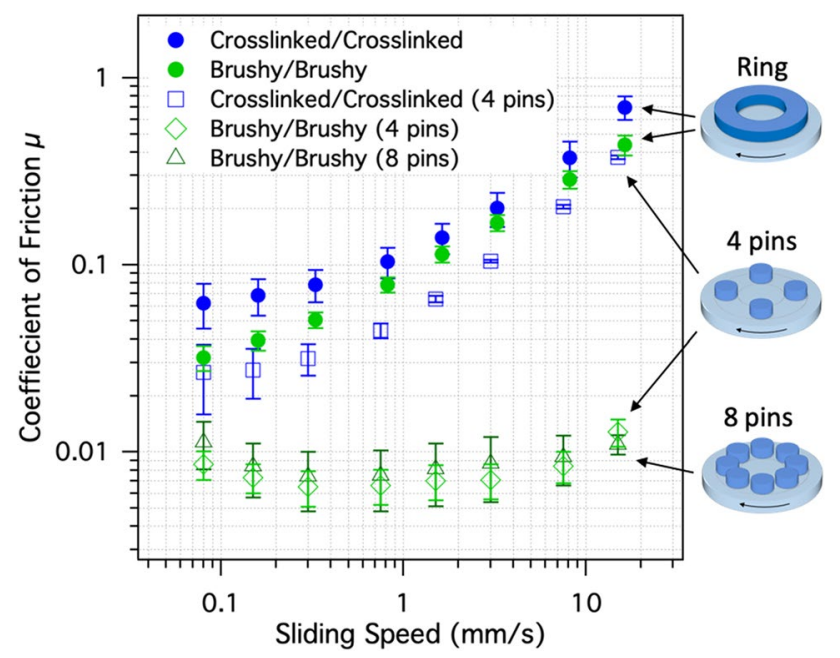

Fig. 4 Coefficient of friction as a function of sliding speed obtained by unidirectional sliding in a ring-on-disc (full symbols) or pins-ondisc configuration (empty symbols). Self-mated, crosslinked contacts are shown in blue and self-mated, brushy contacts are shown in green the sliding speeds. This corresponds to $\lambda$ values of roughly 1 , which in principle does not ensure hydrodynamic lubrication. However, considering the low elastic modulus of these gels, it is likely that the surface asperities flatten under load in order to avoid direct contact between polymer chains, especially when considering bound water layers [28]. Therefore, we assume that a thin but continuous lubrication film is maintained throughout the whole contact area. Moreover, increasing the sliding speed caused an increase in coefficient of friction in a way that could indicate the presence of hydrodynamic effects. Namely, at sliding speeds above $1 \mathrm{~mm} / \mathrm{s}$, the friction coefficient scaled with sliding speed to the power of about 0.6 . Dunn and coworkers performed similar experiments with an aluminum-ring-on-gel setup and, over the same range of sliding speeds, observed that torque increased with sliding speed to the power of $0.86[29,30]$. They attributed the observed sublinear frictional increase to the shear-thinning (thixotropic) behavior of the fluid at the sliding interface. For a hydrogel-hydrogel contact in a ringon-disc configuration, however, the group of Gong observed friction scaling with an exponent of 0.55 [12], which is very similar to our results. Such scaling is close to the theoretically predicted value of 0.5 for hydrodynamic lubrication of soft matter [19, 31]. However, the theory is based on hydrodynamic lift and drag between two non-parallel (i.e., wedge-like) surfaces, as is the case for a pad bearing [32]. In the ring-on-disc experiment, where both sliding surfaces are, in principle, parallel, it might be assumed that no such lubrication should take place. However, there is a possibility that undulations, similar to Schallamach waves, are created during sliding [33], and that these might cause local convergence and divergence of the sliding surfaces, which in turn could lead to soft elasto-hydrodynamic lubrication, as described by Hamrock and Dowson [20]. Moreover, the migrating undulations could also involve dissipation due to poro-elastic flow, which was shown to cause friction scaling with sliding speed to the power of 0.5 [15]. In that study, however, thin hydrogel films and contact pressures above $1 \mathrm{MPa}$ were used, which should be well in excess of the osmotic pressure of the hydrogels used [34]. In our case, the contact pressures were several orders of magnitude lower, and below the assumed osmotic pressure, so dissipation due to interfacial shearing seems to be a more probable mechanism than a poro-elastic mechanism.

When two brushy hydrogel surfaces were slid in a ringon-disc configuration, although the coefficient of friction was indeed lower than in the self-mated crosslinked case, it had a very similar speed-dependent behavior, yielding a slope of about 0.58 . This could be explained by the constant normal stress causing the brushy surfaces to partially exude water, increasing the polymer density and decreasing the permeability in the near-surface region. In this way, initially 
sparse and brushy surfaces begin to behave similarly to the crosslinked ones.

To verify the hypothesis of water exudation, the ring was replaced with four, symmetrically positioned, equally spaced pins, which allowed the disc surface to come out of contact and be exposed to water for a time period approximately equal to the duration of the contact. The normal load was adjusted to match the contact pressure from the experiments with the ring and the tests were performed over the same range of sliding speeds. The results are shown in Fig. 4. For the self-mated, crosslinked case, the coefficient of friction was lower than that of the corresponding ring-on-disc configuration, but maintained almost the same speed dependence. This similarity shows that the leading edge of a flat pin plays no particular role in the friction behavior when compared to a continuous contact. The lower coefficient of friction suggests that the gaps between the pins allowed more water to enter the contact and increase the water film thickness compared to the ring configuration. This might be accompanied by the easier formation of individual wedges in this geometry. For the self-mated, brushy case, however, the coefficient of friction decreased substantially to values between 0.006 and 0.01 showing very little speed dependency. Even when the number of pins was increased to eight, leaving very little gap between them, the friction coefficient remained low and speed independent, as shown in Fig. 4. This suggests that the brushy disc surface could rapidly rehydrate sufficiently in the brief absence of normal and shear stresses.

Figure 5 shows the averaged, steady-state coefficient of friction as a function of sliding speed for the reciprocating sliding of a flat pin over a hydrogel disc. For the self-mated, crosslinked hydrogels, the coefficient of friction increased

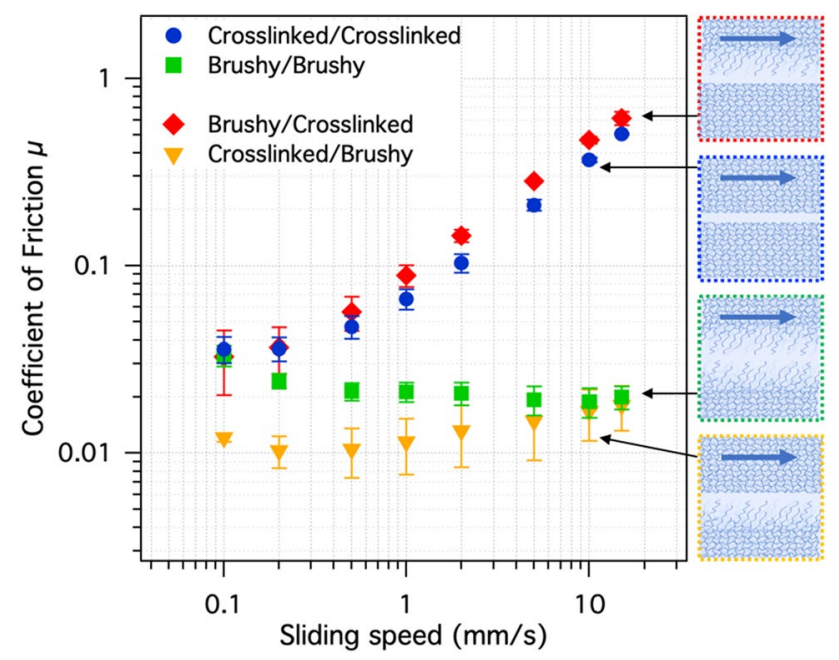

Fig. 5 Coefficient of friction as a function of sliding speed obtained by reciprocating sliding in a flat-pin-on-disc configuration from about 0.03 at a speed of $0.1 \mathrm{~mm} / \mathrm{s}$ to about 0.5 at $15 \mathrm{~mm} / \mathrm{s}$. Above about $1 \mathrm{~mm} / \mathrm{s}$, the friction scaled with the sliding speed raised to the power of $\sim 0.7$. The obtained values and the speed dependency are very similar to the results obtained with the unidirectional sliding of four pins over a disc, Fig. 4. The result is qualitatively also very similar to the results with 7.5 wt.\% PAAm hydrogels obtained using the same reciprocating setup in our previous work [18, 24]. For the self-mated, brushy hydrogels in pin-on-disc contact, the coefficient of friction varied between 0.02 and 0.03 and was almost speed independent, as shown in Fig. 5. The result is again similar to the unidirectional sliding of four pins over a disc, Fig. 4, as well as to our previous results using a similar contact pair [18]. The similarity of the results obtained with unidirectional and reciprocating sliding also shows that the relatively short stroke length, equal to the diameter of the pin, is sufficient to provide contact conditions similar to those encountered in continuous, unidirectional sliding.

Surprisingly, for mismatched hydrogel surfaces in contact, however, the steady-state values of the coefficient of friction for the brushy hydrogel surface sliding over a crosslinked surface were very similar to those in the selfmated crosslinked case, Fig. 5. As discussed below, the constant compressive load and shear stress lead to the exudation of water from the sparse surface region of the brushy hydrogel surface. This results in an increase in the near-surface polymer concentration, with a concomitant drop in permeability and decrease in penetration depth of the shearing liquid. The observed speed-dependent coefficient of friction is thus a consequence of shearing a very thin interfacial layer of water confined between both hydrogel surfaces, similarly to the self-mated crosslinked case.

On the other hand, sliding a crosslinked hydrogel surface over a brushy one resulted in very low, speed-independent friction with values in the range of 0.01-0.02. The observed behavior was similar to that of the self-mated brushy case and presumably originates from the thick water-shearing layer that was provided by the brushy lower specimen. Migration of the contact allowed the brushy surface to reswell during the out-of-contact phase and provide a thick shearing water film during the contact phase. Considerably lower friction values at low sliding speeds compared to the self-mated brushy case are most likely due to the absence of interdigitation of the brushes when one of the sliding partners is crosslinked.

To check if the exudation of water is indeed responsible for the increase in friction as discussed above, the evolution of the coefficient of friction for a brushy hydrogel pin sliding over a crosslinked hydrogel disk was plotted in Fig. 6. The experiments were performed one sliding speed at a time, with sufficient time between the experiments for complete reswelling of the hydrogel surfaces to take place. The coefficient of friction during the first cycle was in the 


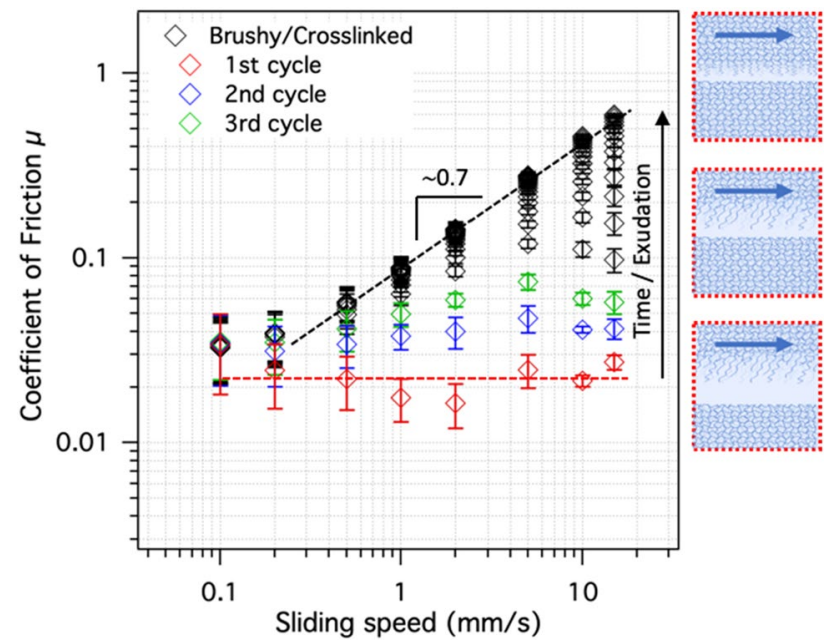

Fig. 6 The evolution of coefficient of friction at each sliding speed during reciprocating sliding of a brushy hydrogel surface over a crosslinked surface. Initially speed-independent friction turned into speed-dependent friction that scaled with speed to the power of $\sim 0.7$. The experiments were performed one sliding speed at a time. The color code depicts the initial sliding cycles at each sliding speed

range of $0.02-0.03$ for all sliding speeds, which is practically identical to the steady-state value of a brushy pin sliding over a brushy surface, Fig. 5 . The friction for a brushy-oncrosslinked case then increased with every cycle, which was more pronounced at high sliding speeds. The coefficient of friction stabilized at the steady-state values presented in Fig. 5, acquiring the characteristic power law scaling with speed $\left(\mu \propto v^{0.7}\right)$. The initial, speed-independent friction in this case indicates that the governing dissipation mechanism is not due to the poro-elastic deformation of the disc. If poro-elastic dissipation within the migrating contact of the disc were the main friction mechanism, the friction would show speed-dependent values from the very beginning of the experiment since it would not be dependent on the hydration of the upper specimen's surface. Therefore, the governing dissipation mechanism indeed seems to be the shearing of the interfacial water. The time-dependent exudation of the interfacial water trapped within the brushy structure reduces the effective shearing thickness, which leads to an increase in friction.

To study the water exudation under load and shear, the time-dependent evolution of coefficient of friction was evaluated. Figure 7 a shows the coefficient of friction as a function of time for all four contact configurations during reciprocating sliding at a nominal speed of $10 \mathrm{~mm} / \mathrm{s}$. The markers correspond to representative experimental data and the solid lines show an exponential fit to the data. The following equation was used to fit the data:
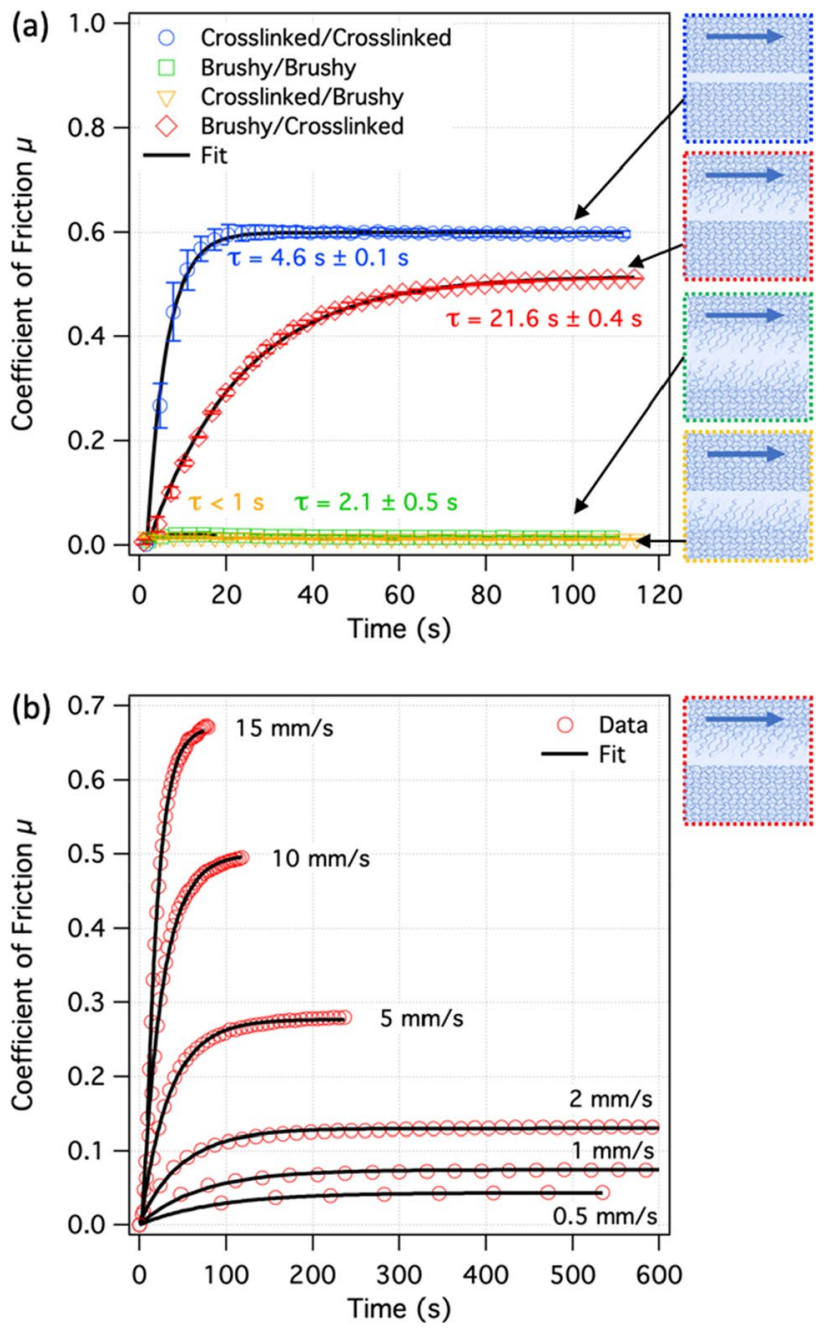

Fig. 7 a Coefficient of friction as a function of time for the four contact configurations during reciprocating sliding at $10 \mathrm{~mm} / \mathrm{s}$ in a flatpin-on-disc configuration. b Coefficient of friction as a function of time during reciprocating sliding of a brushy gel over a crosslinked gel surface at various sliding speeds. An exponential fit was used to extract the characteristic times for friction stabilization

$\mu(t)=\mu_{\infty}-\mu_{1} e^{-t / \tau}$

where $\mu(t)$ is the coefficient of friction at time $t, \mu_{\infty}$ is the equilibrated coefficient of friction, $\mu_{1}$ is the overall change in the coefficient of friction and $\tau$ is the characteristic time until friction stabilization. In both cases with a brushy bottom surface, the friction was low from the very beginning of the sliding test with short characteristic times on the order of 1-2 s. In the self-mated, crosslinked case, the coefficient of friction stabilized at substantially higher values with a relatively short characteristic time of about $5 \mathrm{~s}$. In the brushyon-crosslinked case, however, the time to friction stabilization was about $22 \mathrm{~s}$, which is considerably longer than any other contact combination used in this work. The long stabilization time in this case is attributed to the exudation of 
water from the brushy top surface. To check whether shear has an effect on exudation rate, the coefficient of friction for the brushy-on-crosslinked case was examined at all sliding speeds, Fig. 7b. Exponential fits to the experimental data showed remarkably good agreement and the characteristic times were observed to decrease with increasing sliding speed, Fig. 8. The decreasing of the characteristic times with the increasing sliding speed indicates that the exudation is not driven solely by the normal load, but also by shear, the influence of which is discussed below.

\subsection{Infrared Spectroscopy}

ATR-FTIR spectroscopy was performed to show direct evidence of time-dependent water exudation and consequent
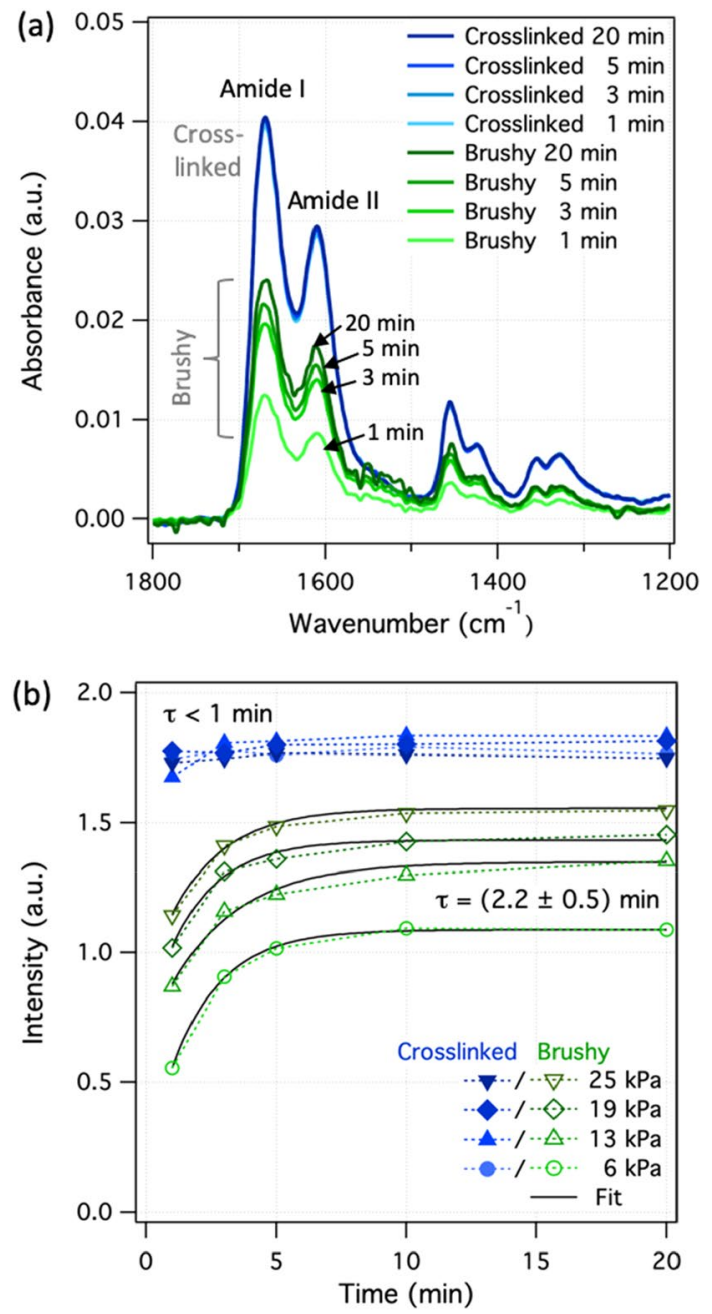

Fig. 8 a Absorption ATR-FTIR spectra of the crosslinked and brushy hydrogel surfaces at various times under a normal pressure of $6 \mathrm{kPa}$. b Intensity of the amide II absorption peak corresponding to the amount of polymer in the near-surface region of the gel as a function of time and normal stress. The full line is the exponential fit to the data that were used to extract the characteristic time $\tau$ polymer densification in the near-surface region of the hydrogel surfaces. Two hydrogel pins of the same dimensions as in the friction experiments, having either a brushy or a crosslinked surface, were pressed against the ATR-FTIR crystal. The spectra obtained at different times after the normal load application are shown in Fig. 8a. The time evolution of the amide II peak intensity as a function of time for both hydrogel surfaces is presented in Fig. 8b. The intensity and therefore the polymer concentration in the near-surface region for the crosslinked hydrogel surface stabilized within the first minute after applying the normal load. Furthermore, the polymer density of the crosslinked hydrogel surfaces did not depend on the normal stress within the tested range of $6-25 \mathrm{kPa}$. This indicates that the tested hydrogels were incompressible at these normal stresses, which agrees with the observation that water cannot be driven out of the gel, assuming that the applied pressure does not exceed the osmotic pressure [34].

On the other hand, the polymer concentration in the nearsurface region of the brushy hydrogels showed substantial time- and normal-stress dependence, Fig. 8b. Increasing the normal stress led to an increase in the polymer concentration, which reflects the densification of the sparse brushy layer, to the point of balance between the osmotic pressure and the external normal stress. To extract the characteristic time for water exudation, the data for the brushy surface in Fig. $8 \mathrm{~b}$ were fitted using an exponential equation similar to Eq. (1). The obtained characteristic times were on the order of $140 \mathrm{~s}$. Surprisingly, no dependence on the normal stress was observed. However, the observed densification could be a consequence of two simultaneous phenomena; vertical redistribution of water within the sparse layer due to its gradient structure, as well as exudation of water out of the contact zone. The latter should be contact-size dependent and will be studied in the near future. Nevertheless, the interplay of the two phenomena might result in time differences that we could not resolve with the applied technique. Therefore, the average time of $(2.2 \pm 0.5)$ min was assumed as the characteristic drainage time under the given stationary conditions.

The characteristic times extracted from the FTIR spectroscopy and from the time-dependent friction increase of the brushy pin sliding over a crosslinked surface are plotted in Fig. 9. Despite the different techniques used and different quantities measured, the obtained characteristic times suggest that the increase in polymer concentration in the surface region is indeed responsible for the increase in friction. The obtained results also show that increasing the sliding speed decreases the characteristic drainage time. Therefore, the exudation seems to be driven by a combination of normal load and shear under the given conditions. We assume that the normal stress and the size of the pin have a significant effect on the drainage times during sliding; however, the 


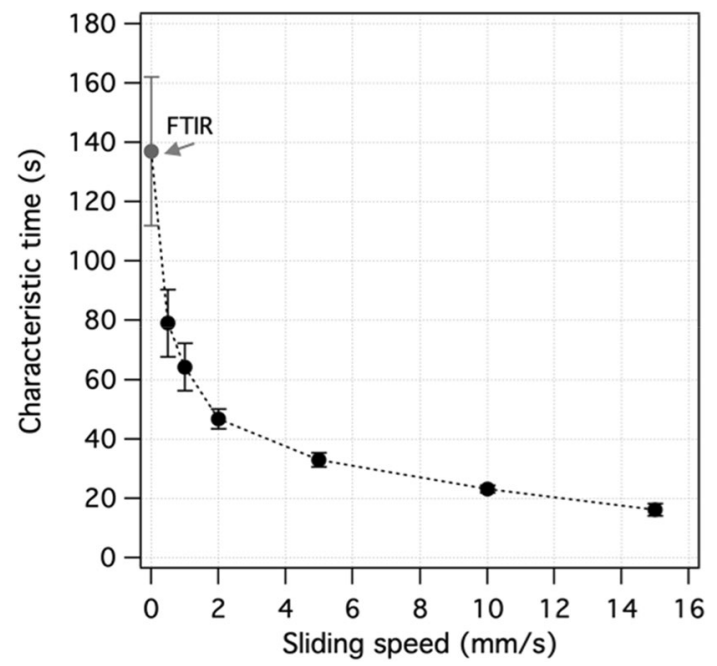

Fig. 9 The characteristic times extracted from the tribological experiments as a function of sliding speed $v$ and from FTIR spectroscopy (at $v=0$ )

effect of the mentioned parameters exceeds the scope of this work and is planned to be studied in the future.

Figure 10 summarizes the contact conditions of various hydrogel surfaces during sliding. The friction of highly solvated, brushy surfaces strongly depends on the contact conditions. Exposing a brushy hydrogel surface to a constant normal stress and shear exudes the water from the brushy layer and thus decreases the thickness of the interfacial shearing layer, Fig. 10a-c. This results in a friction that scales with sliding speed as $\mu \propto v^{0.5-0.7}$, similarly to the behavior of the self-mated crosslinked case. If a brushy surface is only temporarily exposed to normal load and shear, it is able to rehydrate during the out-of-contact phase,
Figs. 10d-f. In this case, a balance between exudation and rehydration is maintained, enabling a thicker shearing film, similar to that seen for the self-mated contact of brushy surfaces, Fig. 10f. The thick shearing water layer reduces the shear stress and therefore the frictional force. While the proposed mechanisms are based on results obtained with flat-on-flat geometry, preliminary results indicate that they also hold for sphere-on-flat geometry within the tested range of sliding speeds. At higher sliding speeds, however, a convergent zone in the sphere-on-flat geometry might facilitate the entrainment of water and thus reduce friction. This will be the topic of future study.

\section{Conclusion}

PAAm hydrogels with either brushy or crosslinked surfaces were prepared by polymerization against two different molding surfaces. Tribological experiments showed that two crosslinked hydrogels in a sliding contact have a speed-dependent friction, irrespective of the contact geometry used. The self-mated crosslinked acrylamide hydrogels always showed friction that increased with sliding speed to the power of 0.5-0.7, whether in ring-on-disc or pin-on-disc configuration, in unidirectional motion or under reciprocating sliding. This is attributed to hydrodynamic lubrication, where a very thin, nanoscopic water film is being sheared between both hydrogel surfaces.

For the sparse and brushy acrylamide hydrogel surfaces, however, the friction depends on their ability to rehydrate. Brushy surfaces exposed to a constant normal load and shear partially exuded water over time, which increased the polymer concentration in the near-surface region. This resulted in friction behavior that was similar to that of self-mated

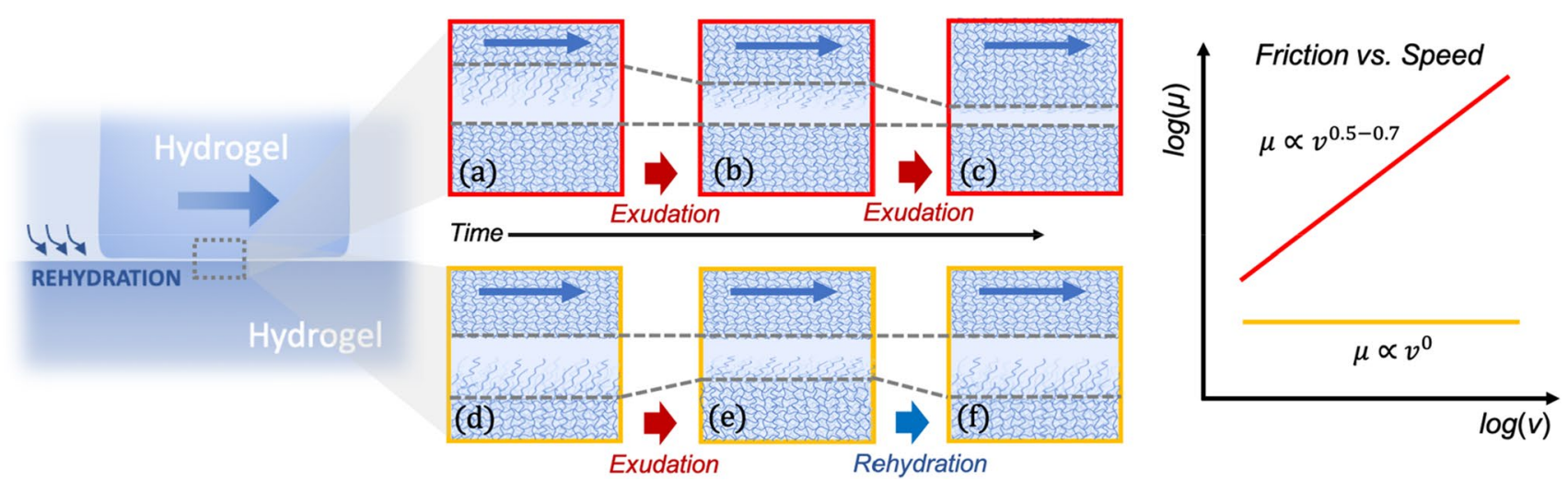

Fig. 10 Schematic of contact conditions and resulting friction. An initially thick water layer in a brushy-on-crosslinked contact (a) is exuded under contact (b). Continuous compression and shear finally result in a thin shearing film (c), which is similar to the selfmated crosslinked case and causes speed-dependent friction. The crosslinked-on-brushy contact (d) is also partially exuded under contact (e). The rehydration during the out-of-contact phase recovers the film thickness (f), which results in speed-independent friction, similarly to the brushy-brushy contact 
crosslinked surfaces, where the water-shearing region is limited to a very thin interfacial film. This occurred in the case of a brushy hydrogel pin sliding over a crosslinked surface as well as in the case of brushy surfaces in a ring-on-disc configuration. In both these cases, no time for rehydration of the brushy surfaces was available. On the other hand, allowing the brushy surfaces to rehydrate during an out-ofcontact time moves the system into the low-friction, speedindependent regime. According to the results in this work, the rehydration times for acrylamide hydrogels seem to be shorter than the exudation times, which were on the order of minutes under normal stress alone and decreased when the shear was applied. The obtained results also indicate the importance of contact design, in order to exploit the lowfriction properties of brushy surfaces.

The results in this work also show that the dissipation mechanism underlying speed-dependent friction between acrylamide hydrogels is due to the hydrodynamic shearing of a thin interfacial water layer rather than poro- or viscoelastic dissipation related to the migrating deformation. Deformation-induced poro-elastic flow, however, could become significant at higher contact pressures that are able to overcome the osmotic pressure of the stressed hydrogel. However, more experiments are needed to determine the contact conditions under which such a mechanism would come into play.

Acknowledgements This project has received funding from the European Research Council (ERC) under the European Union's Horizon 2020 research and innovation programme (Grant Agreement No 669562). The authors would also like to express their gratitude to Dr. André Brem for help with the rheometer setup and to Dr. Andrea Arcifa for fruitful discussions about hydrodynamic lubrication.

Open Access This article is licensed under a Creative Commons Attribution 4.0 International License, which permits use, sharing, adaptation, distribution and reproduction in any medium or format, as long as you give appropriate credit to the original author(s) and the source, provide a link to the Creative Commons licence, and indicate if changes were made. The images or other third party material in this article are included in the article's Creative Commons licence, unless indicated otherwise in a credit line to the material. If material is not included in the article's Creative Commons licence and your intended use is not permitted by statutory regulation or exceeds the permitted use, you will need to obtain permission directly from the copyright holder. To view a copy of this licence, visit http://creativecommons.org/licenses/by/4.0/.

\section{References}

1. Bansil, R., Stanley, E., LaMont, J.T.: Mucin biophysics. Annu. Rev. Physiol. 57, 635-657 (1995)

2. Lee, S., Spencer, N.D.: Sweet, hairy, soft, and slippery. Science 319, 575-576 (2008)

3. Crockett, R., Grubelnik, A., Roos, S., Dora, C., Born, W., Troxler, H.: Biochemical composition of the superficial layer of articular cartilage. J. Biomed. Mater. Res. Part A 82, 958-964 (2007)

4. Pitenis, A.A., Sawyer, W.G.: Lubricity of high water content aqueous gels. Tribol. Lett. 66, 113 (2018)
5. Gong, J., Higa, M., Iwasaki, Y., Katsuyama, Y., Osada, Y.: Friction of gels. J. Phys. Chem. B 101, 5487-5489 (1997)

6. Gong, J., Osada, Y.: Gel friction: a model based on surface repulsion and adsorption. J. Chem. Phys. 109, 8062-8068 (1998)

7. Gong, J., Iwasaki, Y., Osada, Y., Kurihara, K., Hamai, Y.: Friction of gels. 3. Friction on solid surfaces. J. Phys. Chem. B 103, 6001-6006 (1999)

8. de Gennes, P.G.: Scaling Concepts in Polymer Physics. Cornell University Press, Ithaca (1979)

9. Urueña, J.M., Pitenis, A.A., Nixon, R.M., Schulze, K.D., Angelini, T.E., Sawyer, W.G.: Mesh size control of polymer fluctuation lubrication in gemini hydrogels. Biotribology 1, 24-29 (2015)

10. Gong, J.P., Kagata, G., Osada, Y.: Friction of gels. 4. Friction on charged gels. J. Phys. Chem. B 103, 6007-6014 (1999)

11. Gong, J.P., Iwasaki, Y., Osada, Y.: Friction of gels. 5. Negative load dependence of polysaccharide gels. J. Phys. Chem. B 104, 34233428 (2000)

12. Kagata, G., Gong, J.P., Osada, Y.: Friction of gels. 6. Effects of sliding velocity and viscoelastic responses of the network. J. Phys. Chem. B 106, 4596-4601 (2002)

13. Kagata, G., Gong, J.P., Osada, Y.: Friction of gels. 7. Observation of static friction between like-charged gels. J. Phys. Chem. B 107, 10221-10225 (2003)

14. Pitenis, A., Urueña, J., Schulze, K., Nixon, R., Dunn, A., Krick, B., Sawyer, W., Angelini, T.: Polymer fluctuation lubrication in hydrogel gemini interfaces. Soft Matter 10, 8955-8962 (2014)

15. Delavoipière, J., Tran, Y., Verneuil, E., Heurtefeu, B., Hui, C.Y., Chateauminois, A.: Friction of poroelastic contacts with thin hydrogel films. Langmuir 34, 9617-9626 (2018)

16. Delavoipière, J., Tran, Y., Verneuil, E., Chateauminois, A.: Poroelastic indentation of mechanically confined hydrogel layers. Soft Matter 12, 8049-8058 (2016)

17. Gong, J.P., Kurokawa, T., Narita, T., Kagata, G., Osada, Y., Nishimura, G., Kinjo, M.: Synthesis of hydrogels with extremely low surface friction. J. Am. Chem. Soc. 123, 5582-5583 (2001)

18. Meier, Y.A., Zhang, K., Spencer, N.D., Simic, R.: Linking friction and surface properties of hydrogels molded against materials of different surface energies. Langmuir 35, 15805-15812 (2019)

19. Martin, A., Clain, J., Buguin, A., Brochard-Wyart, F.: Wetting transitions at soft, sliding interfaces. Phys. Rev. E 65, 031605 (2002)

20. Hamrock, B.J., Dowson, D.: Elastohydrodynamic lubrication of elliptical contacts for materials of low elastic modulus I-fully flooded conjunction. J. Lubr. Technol. 100, 236-245 (1978)

21. Moore, A., Burris, D.: Tribological rehydration of cartilage and its potential role in preserving joint health. Osteoarthr. Cartil. 25, 99-107 (2017)

22. Zhang, K., Simic, R., Yan, W., Spencer, N.D.: Creating an interface: rendering a double-network hydrogel lubricious via spontaneous delamination. ACS Appl. Mater. Interfaces 11, 25427-25435 (2019)

23. Kii, A., Xu, J., Gong, J.P., Osada, Y., Zhang, X.: Heterogeneous polymerization of hydrogels on hydrophobic substrate. J. Phys. Chem. B 105, 4565-4571 (2001)

24. Gombert, Y., Simič, R., Roncoroni, F., Dübner, M., Geue, T., Spencer, N.D.: Structuring hydrogel surfaces for tribology. Adv. Mater. Interfaces 6, 1901320 (2019)

25. Sader, J.E., Chon, J.W., Mulvaney, P.: Calibration of rectangular atomic force microscope cantilevers. Rev. Sci. Instrum. 70, 39673969 (1999)

26. Cannara, R.J., Eglin, M., Carpick, R.W.: Lateral force calibration in atomic force microscopy: a new lateral force calibration method and general guidelines for optimization. Rev. Sci. Instrum. 77, 053701 (2006)

27. Lu, X., Mi, Y.: Characterization of the interfacial interaction between polyacrylamide and silicon substrate by Fourier transform infrared spectroscopy. Macromolecules 38, 839-843 (2005) 
28. Raviv, U., Laurat, P., Klein, J.: Fluidity of water confined to subnanometre films. Nature 413, 51-54 (2001)

29. Kim, J., Dunn, A.C.: Soft hydrated sliding interfaces as complex fluids. Soft Matter 12, 6536-6546 (2016)

30. Kim, J., Dunn, A.C.: Thixotropic mechanics in soft hydrated sliding interfaces. Tribol. Lett. 66, 102 (2018)

31. de Gennes, P.G., Brochard-Wyart, F., Quéré, D.: Capillarity and Wetting Phenomena: Drops, Bubbles, Pearls Waves. Springer, New York (2013)

32. Cameron, A.: Basic Lubrication Theory. Ellis Horwood Ltd., London (1981)
33. Schallamach, A.: How does rubber slide? Wear 17, 301-312 (1971)

34. Schulze, K.D., Hart, S.M., Marshall, S.L., O’Bryan, C.S., Urueña, J.M., Pitenis, A.A., Sawyer, W.G., Angelini, T.E.: Polymer osmotic pressure in hydrogel contact mechanics. Biotribology 11, 3-7 (2017)

Publisher's Note Springer Nature remains neutral with regard to jurisdictional claims in published maps and institutional affiliations. 